\title{
Quiste neuroentérico supratentorial: reporte de un caso
}

\author{
Pedro Vázquez Soto ${ }^{1}$, Arturo Ruiz-Aburto A², Alfredo Yáñez Lermanda ${ }^{1}$, Facundo las Heras ${ }^{3}$, Gonzalo Miranda ${ }^{4}$ \\ 1 Neurocirujano, Hospital Clínico Universidad de Chile. \\ 2 Becado neurocirugía, Hospital Clínico Universidad de Chile. \\ 3 Neuropatologo, Departamento de Radiología, Hospital Clínico Universidad de Chile. \\ 4 Neuroparadiologo, Departamento de Anatomía Patológica, Hospital Clínico Universidad de Chile.
}

Rev. Chil. Neurocirugía 44: 48-51, 2018

\section{Resumen}

El quiste neuroentérico es una lesión benigna poco frecuente, de típica ubicación espinal. Su presentación intracranial es rara y más aún su presentación supratentorial. Se presenta el caso de un paciente masculino de 53 años, que en el estudio por cefalea se diagnostica una lesión quistica, que inicialmente se maneja con observación y que a 5 años de seguimiento presenta crecimiento, indicándose manejo quirúrgico, lográndose una resección total de la lesión, cuya biopsia es compatible con un quiste neuroentérico. Seguimiento por 8 años, sin recidiva. Se discute esta entidad como parte del diagnostico diferencial de otras lesiones supratentoriales y de la importancia de la resección total que determina los buenos resultados.

Palabras clave: Quiste neuroentérico, quiste enterogénico, tumor quistico.

\begin{abstract}
The neurenteric cyst is a rare benign lesion injury, with a spinal frecuent location. Intracranial presentation is rare and even more supratentorial. We related the case of a male patient of 53 years wirh a history of headache, that the study show a cystic lesion, initially managed with observation and that 5 years later shows growth, indicating the surgical management, achieving total resection. The biopsy was consistent with a neurenteric cyst. The patient was tracked for 8 years without recurrence. this entity is discussed as part of the differential diagnosis of other supratentorial lesions and the importance of total resection that determines the good outcome
\end{abstract}

Key words: Neuroenteric cyst, enterogenic cyst, cystic tumours.

\section{Introducción}

El quiste neuroentérico es una lesión endodérmica benigna muy infrecuente, que en la mayoría de los casos su ubicación es espinal ${ }^{1-2}$. Su presentación intracranial se presenta más comúnmente en la fosa posterior (Prepontina, cerebelomedular o en el angulo pontocerebeloso) ${ }^{3}$. Excepcionalmente, esta descrito como una lesión supratentorial. Se reporta el caso de un paciente con una lesión en esta ubicación.

\section{Reporte del caso}

Se presenta el caso de un paciente masculino de 53 años de edad, con el único antecedente de asma bronquial en tratamiento médico, que presenta una historia de larga data de cefalea, sin déficit neurológico, contexto en el que se realiza una tomografía computada de cerebro que identifica una lesión quística supratentorial. Se complementa el estudio con Resonancia magnética (RM) de cerebro con con- traste (Figura 1) que evidencia una lesión supratentorial quística frontal izquierda, extra-axial de unos $3 \mathrm{~cm}$ de diámetro mayor, isointensa al líquido cefalorraquídeo en las secuencias T1 y T2. Hiperintensa en FLAIR y no restringe en difusión. Produce desplazamiento de parénquima adyacente, sin signos de gliosis y sin comunicación con el sistema ventricular, que impresiona ser compatible con un quiste aracnoidal. Además se diagnostica una lesión selar, compatible con un macroadenoma hipofisiario, compatible con exámenes de laboratorio con un Prolactino- 


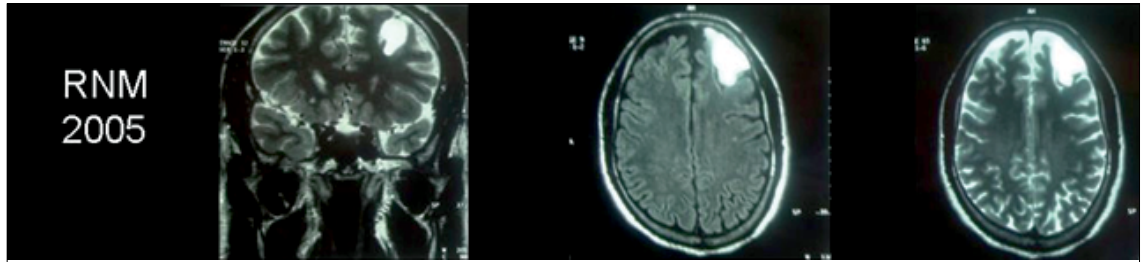

Figura 1. RNM de cerebro lesión supratentorial quística frontal izquierda, extra-axial de unos $3 \mathrm{~cm}$ de diámetro mayor, isointensa al líquido cefalorraquídeo en las secuencias T1 y T2. Hiperintensa en FLAIR y no restringe en difusión. Se considera quiste aracnoidal.

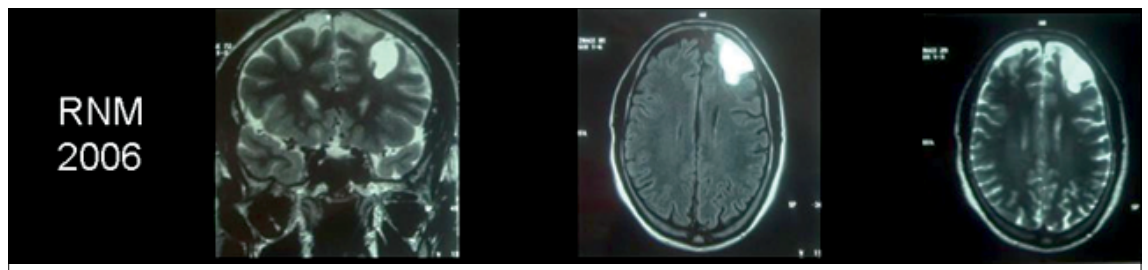

Figura 2. RNM de cerebro un año posterior a primer control, sin cambios con respecto a estudio previo.

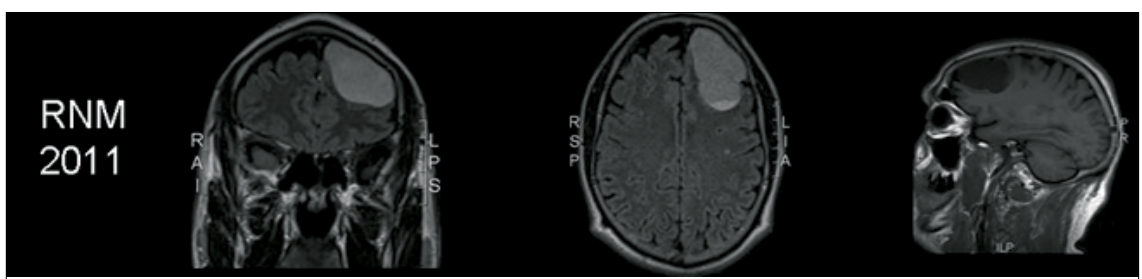

Figura 3. Se evidencia aumento de tamaño de lesión quística, sospechosa de quiste neuroepitelial o glioma de bajo grado quístico.

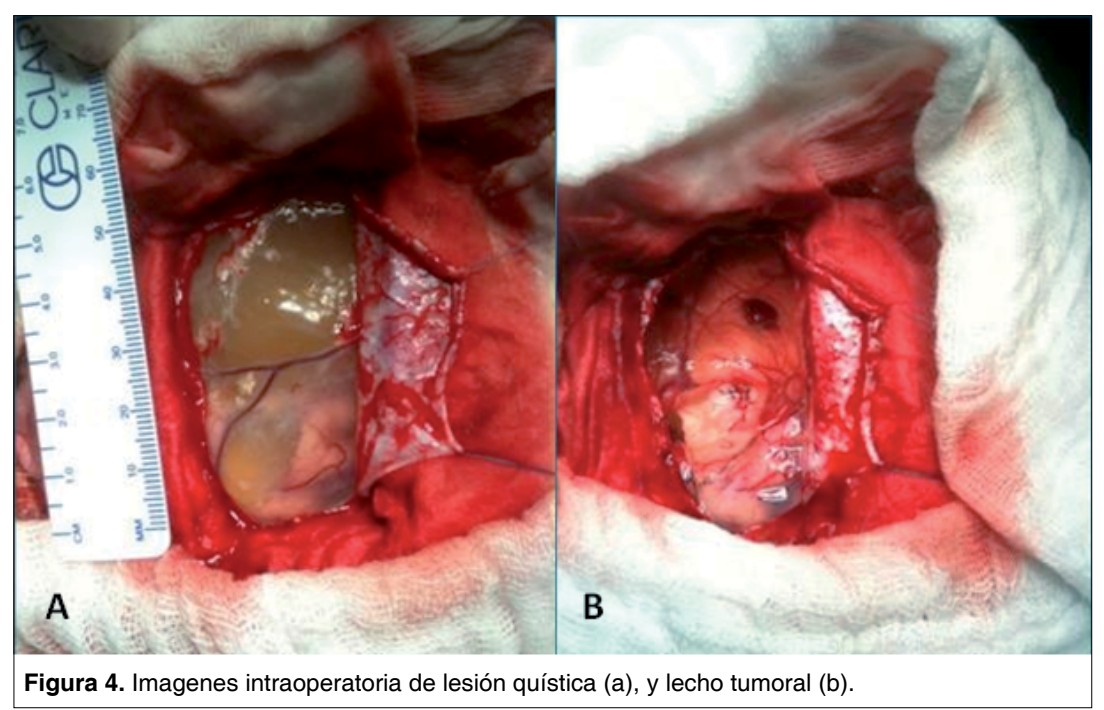

ma, que se maneja medicamente. Con estas medidas, el paciente no presenta síntomas y en el control con Resonancia magnética al año (Figura 2), no muestra cambios de la lesión quística, por lo que se decide la observación clínica. Cinco años después del diagnóstico inicial, presenta nuevamente cefalea acompañado de apatía, sin otras alteraciones neurológicas, controlándose con RM de cerebro (Figura 3), mostrando un importante aumento de tamaño de la lesión quística frontal izquierda, de mas de $5 \mathrm{~cm}$ de diámetro, pero con las mismas características previas, planteándose como diagnóstico diferencial un quiste neuroglial o un tumor glial con componente quístico. En base a esta sospecha, se decide la resección quirúrgica transcraneal de la lesión, por una craneotomía frontal izquierda. Durante la cirugía se observa lesión quística tabicada de aproximadamente 5 por $5,3 \mathrm{~cm}$ (Figura 4a), con una delgada capsula transparente y contenido heterogéneo bifásico, con bordes netos, sin invasión de la duramadre ni del parénquima circundante, compuesto por un líquido seroso y una sustancia gelatinosa amarillo-verdosa, que precipitaba en el fondo del quiste. Se extrae contenido y capsula de la lesión, logrando exéresis total (Figura 4b).

Se efectúa el procedimiento sin incidentes, con estadía postoperatoria en la unidad de cuidados intensivos, sin trastornos hemodinámicos ni hidroelectrolíticos y sin presentar ningún tipo de déficit neurológico, con control postoperatorio con TAC de cerebro sin contraste que solo evidencia un pequeño pneumoencefalo, sin evidencia de complicaciones (Figura 5a). A las 72 horas después del procedimiento paciente se maneja en sala y al quinto día se decide el alta médica. Se programa control con TAC de cerebro al mes del alta, que se aprecia sin complicaciones y con reexpansión de parénquima perilesional (Figura 5b). La anatomía patológica describió una lesión con pared quística revestida de una monocapa epitelial con gran cantidad de células caliciformes que presenta reactividad a la tinción inmunohistoquimica con EMA (Figura 6), característico de revestimiento epitelial, siendo concordantes los hallazgos con un quiste neuroenterico. Paciente no recibió ningún tipo de manejo coadyuvante y a 4 años de la cirugía el paciente se encuentra en buenas condiciones, asintomático y con controles que no evidencian ningún tipo de recidiva local.

\section{Discusión}

Las lesiones quísticas intracraneales en general son infrecuentes e incluyen diversas entidades patológicas, como quistes aracnoideos, coloideos, dermoides, neuroepiteliales, tumores 


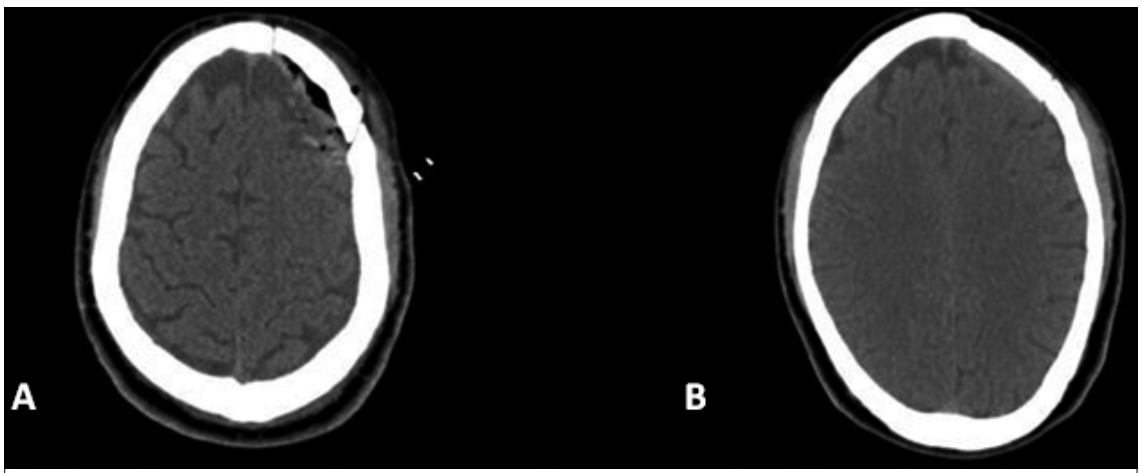

Figura 5. TAC de cerebro post-operatorio: pequeño pneumoencefalo (a), Tac al mes post-operatorio con reexpanción de parenquima (b).

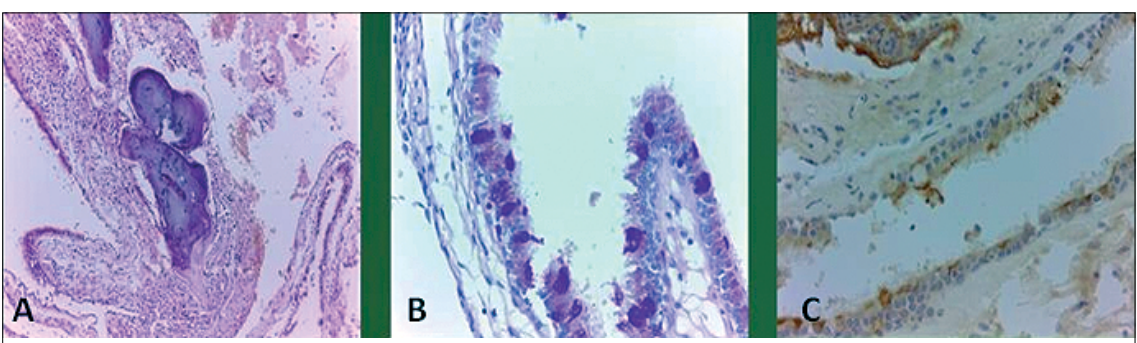

Figura 6. Anatomía patológica: Pared quística revestida por monocapa epitelial (a), células caliciformes con tinción de PAS (b), Tinción Inmunohistoquimica EMA, evidenciando revestimiento epitelial (c).

con componente quístico, parásitos, entre los más señalados en la literatu$\mathrm{ra}^{1,2,3,4,6,7}$. Descrito por Puusepp et al., en el año 1934, como un intestinoma. El quiste neuroentérico, es una lesión benigna malformativa poco frecuente, compuesta por elementos endodérmicos localizados en el sistema nervioso central, que en su aspecto histológico semejan a la mucosa gastrointestinal (también conocidos como quistes enterógenos o gastrogénicos) $)^{1}$. Su origen exacto es desconocido. Probablemente surgen en el desarrollo notocorda durante la existencia transitoria del canal neuroentérico. La notocorda y el intestino anterior fallarían en separarse, por lo que células primitivas endodérmicas se incorporarían a la notocorda, Con respecto a su ubicación, la mayoría se ubican a nivel espinal, siendo su localización intracraneal rara, descrita en menos del $25 \%$ de los casos (alrededor de 60 casos publicados). De estos, el $75 \%$ se ubican en la fosa posterior en relación a la línea media frente a la unión pontobulbar. De los intracraniales solo el $25 \%$ son supratentoriales, de ubicación fuera de la línea media adyacentes a los lóbulos frontales ${ }^{11}$. Se presentan como quistes extraxiales lisos, de paredes delgadas y contenido variable, desde líquido transparente a contenido espeso mucoide, con un ta- maño variable, que habitualmente < es menor a $3 \mathrm{~cm}$. En los supratentoriales se han descrito de mayor tamaño, alcanzando hasta $9 \mathrm{~cm}^{2,8}$.

Por su origen endodérmico, la anatomía patológica describe una lesión que presenta un epitelio que puede representar a los distintos precursores del endodermo, desde un epitelio ciliado, cuboidal, columnar tipo respiratorio o intestinal y también secretor de muci$\mathrm{na}^{11}$. En general, se comportan como lesiones de bajo potencial proliferativo y es excepcional que presenten una lesión maligna, describiéndose en esos casos la formación de adenocarcinomas $^{7}$. Clínicamente se presentan con síntomas inespecíficos como cefalea, pero pueden provocar clínica por el efecto de masa donde se encuentren, crisis convulsivas, hidrocefalia (en ubicaciones paraventriculares) y meningitis aséptica ${ }^{5,13,14,21}$. El tratamiento de elección de esta lesión es la exéresis quirúrgica, medida que tiene muy buenos resultados en la mayoría de los casos descritos. Estos quistes pueden recidivar, por lo que la resección total debe lograrse en todos los casos, objetivo que se logra con la resección completa de la pared del quiste ${ }^{6}$. No se han descrito tratamientos alternativos a la cirugía ni tampoco terapia coadyuvantes para disminuir la recidiva ${ }^{1,9,11}$.

\section{Conclusión}

El quiste neuroentérico es una entidad benigna, si bien su presentación intracranial-infratentorial es la más frecuente, es bueno tenerlo como diagnóstico diferencial de lesión quística supratentorial.

\section{Recibido: 21 de agosto de 2017} Aceptado: 22 de septiembre de 2017

\section{Referencias}

1. Gauden AJ, Khurana VG, Tsui AE, Kaye AH. Intracranial neuroenteric cysts: a concise review including an illustrative patient. $\mathrm{J}$ Clin Neurosci. 2012 Mar;19(3):352-9.

2. Osborn AG, Preece MT. Intracranial cysts: Radiologic -pathologic correlation and imaging approach. Radiology. 2006;239:650-4.

3. Bejjani GK, Wright CD, Schessel D, Sekhar LN. Endodermal cysts of the posterior fossa. J Neurosurg. 1998;29:893-7.

4. Preece MT, Osborn AG, Chenn SS. Intracranial neurenteric cysts: Imaging and pathology spectrum. AJNR Am J Neuroradiol. 2006;27:1211-6.

5. Achard JM, Lallement PY, Veyssier P. Recurrent aseptic meningitis secondary to intracranial epidermoid cyst and Mollaret meningitis: Two distinct entities or a single disease. A case report and nosologic discussion? Am J Med. 1990;89:807-10. 
6. Chen CT, Lee CY, Lee ST, Chang CN, Wei KC, Wu CT. Neurenteric cysts: risk factors and management of recurrence. Acta Neurochir (Wien). 2016 Jul;158(7):1325-31.

7. Yang Y, Fang J, Li DA, Wang L, Ji N, Zhang J. Recurrent intracranial neurenteric cyst with malignant transformation: A case report and literature review. See comment in PubMed Commons belowOncol Lett. 2016 May;11(5):3395-3402.

8. Medhi G, Saini J, Pandey P, Mahadevan A, Prasad C. T1 Hyperintense Prepontine Mass with Restricted Diffusion-A White Epidermoid or a Neuroenteric Cyst?. J Neuroimaging. 2015 Sep-Oct;25(5):841-3.

9. Breshears JD, Rutkowski MJ, McDermott MW, Cha S, Tihan T, Theodosopoulos PV. Surgical Management of Intracranial Neuroenteric Cysts: The UCSF Experience.J Neurol Surg B Skull Base. 2015 Dec;76(6):475-9.

10. Prasad GL, Sharma BS, Mahapatra AK. Ventral foramen magnum neurenteric cysts: a case series and review of literature. Neurosurg Rev. 2015 Dec 10.

11. Chakraborty S, Priamo F, Loven T, Li J, Insinga S, Schulder M. Supratentorial Neurenteric Cysts: Case Series and Review of Pathology, Imaging, and Clinical Management. World Neurosurg. 2016 Jan;85:143-52.

12. Junaid M, Kalsoom A, Khalid M, Bukhari SS. Giant supratentorial neurenteric cyst. J Coll Physicians Surg Pak. 2014 Nov;24 Suppl 3:S214-5.

13. Miller CM, Wang BH, Moon SJ, Chen E, Wang H. Neurenteric cyst of the area postrema. Case Rep Neurol Med. 2014;2014:718415.

14. Choh NA, Wani M, Nazir P, Saleem SM, Shaheen F, Rabbani I, Gojwari T. Intracranial neurenteric cyst: A rare cause of chemical meningitis. Ann Indian Acad Neurol. 2013 Apr;16(2):286-8.

15. Kitamura Y, Sasaki H, Hashiguchi A, Momoshima S, Shidoh S, Yoshida K. Supratentorial neurenteric cyst with spontaneous repetitive intracystic hemorrhage mimicking brain abscess: a case report. Neurosurg Rev. 2014 Jan;37(1):153-9.

16. Little MW, Guilfoyle MR, Bulters DO, Scoffings DJ, O'Donovan DG, Kirkpatrick PJ. Neurenteric cyst of the anterior cranial fossa: case report and literature review. Acta Neurochirurgica. 2011;153(7):1519.1525.

17. Miyagi A, Katayama Y. Neurenteric cyst arising in the high convexity parietal lesion: case report. Neurosurgery. 2007;60(1):E203-E204.

18. Preece MT, Osborn AG, Chin SS, Smirniotopoulos JG. Intracranial neurenteric cysts: imaging and pathology spectrum. American Journal of Neuroradiology. 2006;27(6):1211-1216.

19. Teufack S, Campbell P, Moshel Y. Intracranial neurenteric cysts: two atypical cases and review of the literature. Jefferson Hospital for Neuroscience Journal. 2011;6(1):18-

20. Wang L, Zhang J, Wu Z, et al. Diagnosis and management of adult intracranial neurenteric cysts. Neurosurgery. 2011;68(1):44-52.

21. Marchionni M, Smith C, Eljamel MS. Intracranial enterogenous cyst extending into both supratentorial and infratentorial compartments: case report and review of the literature. Skull Base. 2008;18(3):213-216.

\section{Correspondencia:}

Dr. Pedro Vázquez Soto

Serv. Neurocirugía, Hospital Clínico Universidad de Chile.

pvazquezs@yahoo.es 